\title{
Some Results on Characterizations of Matrix Partial Orderings
}

\author{
Hongxing Wang and Jin Xu \\ Department of Mathematics, Huainan Normal University, Anhui 232001, China \\ Correspondence should be addressed to Jin Xu; xujin_1986hn@163.com
}

Received 19 February 2014; Accepted 21 April 2014; Published 28 May 2014

Academic Editor: Yang Zhang

Copyright (C) $2014 \mathrm{H}$. Wang and J. Xu. This is an open access article distributed under the Creative Commons Attribution License, which permits unrestricted use, distribution, and reproduction in any medium, provided the original work is properly cited.

\begin{abstract}
Some characterizations of the left-star, right-star, and star partial orderings between matrices of the same size are obtained. Based on those results, several characterizations of the star partial ordering between EP matrices are given. At last, one characterization of the sharp partial ordering between group matrices is obtained.
\end{abstract}

\section{Introduction}

In this paper we use the following notation. Let $\mathbb{C}^{m \times n}$ be the set of complex $m \times n$ matrices. For any matrix $A \in \mathbb{C}^{m \times n}, A^{*}$, $\mathfrak{R}(A)$, and $r(A)$ denote the conjugate transpose, the range, and the rank of $A$, respectively. The symbol $I_{n}$ denotes the $n \times$ $n$ identity matrix, and 0 denotes a zero matrix of appropriate size. The Moore-Penrose inverse of a matrix $A \in \mathbb{C}^{m \times n}$, denoted by $A^{\dagger}$, is defined to be the unique matrix $X \in \mathbb{C}^{n \times m}$ satisfying the four matrix equations

$$
\begin{aligned}
& \text { (1) } A X A=A \\
& \text { (2) } X A X=X \\
& \text { (3) }(A X)^{*}=A X \\
& \text { (4) }(X A)^{*}=X A
\end{aligned}
$$

and $A^{-}$denotes any solution to the matrix equation $A X A=$ $A$ with respect to $X ; A\{1\}$ denotes the set of $A^{-}$; that is, $A\{1\}=$ $\{X \mid A X A=A\}$. Moreover, $A^{\#}$ denotes the group inverse of $A$ with $r\left(A^{2}\right)=r(A)$, that is, the unique solution to

$$
\begin{aligned}
& \text { (1) } A X A=A, \\
& \text { (2) } X A X=X, \\
& \text { (5) } A X=X A .
\end{aligned}
$$

It is well known that $A^{\#}$ exists if and only if $r\left(A^{2}\right)=r(A)$, where case $A$ is also called a group matrix. A matrix $A$ is EP if and only if $A$ is a group matrix with $A^{\#}=A^{\dagger}$. The symbols $\mathbb{C}_{\mathrm{GP}}^{n}$ and $\mathbb{C}_{\mathrm{EP}}^{n}$ stand for the subset of $\mathbb{C}^{n \times n}$ consisting of group matrices and EP matrices, respectively (see, e.g., $[1,2]$ for details).

Five matrix partial orderings defined in $\mathbb{C}^{m \times n}$ are considered in this paper. The first of them is the minus partial ordering defined by Hartwig [3] and Nambooripad [4] independently in 1980:

$$
A \leq B \Longleftrightarrow A^{-} A=A^{-} B, \quad A A^{=}=B A^{=},
$$

where $A^{-}, A^{=} \in A\{1\}$. In [3] it was shown that

$$
A \leq B \Longleftrightarrow r(B-A)=r(B)-r(A) \text {. }
$$

The rank equality indicates why the minus partial ordering is also called the rank-subtractivity partial ordering. In the same paper [3] it was also shown that

$$
A \stackrel{*}{\leq} B \Longleftrightarrow r\left[\begin{array}{l}
A \\
B
\end{array}\right]=r\left[\begin{array}{ll}
A & B
\end{array}\right]=r(B), \quad A B^{-} A=A,
$$

where $B^{-} \in B\{1\}$.

The second partial ordering of interest is the star partial ordering introduced by Drazin [5], which is determined by

$$
A \stackrel{*}{\leq} B \Longleftrightarrow A^{\dagger} A=A^{\dagger} B, \quad A A^{\dagger}=B A^{\dagger} .
$$

It is well known that

$$
A \stackrel{*}{\leq} B \Longleftrightarrow A^{*} A=A^{*} B, \quad A A^{*}=B A^{*} .
$$


In 1991, Baksalary and Mitra [6] defined the left-star and right-star partial orderings characterized as

$$
\begin{gathered}
A^{*} \leq B \Longleftrightarrow A^{*} A=A^{*} B, \quad \mathfrak{R}(A) \subseteq \mathfrak{R}(B), \\
A \leq^{*} B \Longleftrightarrow A A^{*}=B A^{*}, \quad \mathfrak{R}\left(A^{*}\right) \subseteq \mathfrak{R}\left(B^{*}\right) .
\end{gathered}
$$

The last partial ordering we will deal with in this paper is the sharp partial ordering, introduced by Mitra [7] in 1987, and is defined in the set $\mathbb{C}_{\mathrm{GP}}^{n}$ by

$$
A \leq^{\#} B \Longleftrightarrow A^{\#} A=A^{\#} B, \quad A A^{\#}=B A^{\#} .
$$

A detailed discussion of partial orderings and their applications can be found in $[1,8-10]$.

It is well known that rank of matrix is an important tool in matrix theory and its applications, and many problems are closely related with the ranks of some matrix expressions under some restrictions (see [11-15] for details). Our aim in this paper is to characterize the left-star, right-star, star, and sharp partial orderings by applying rank equalities. In the following, when $A$ is considered below $B$ with respect to one partial ordering, then the partial ordering should entail the assumption $r(A)>r(B) \geq 1$.

\section{The Star Partial Ordering}

Let $A$ and $B$ be $m \times n$ complex matrices with ranks $a$ and $b$, respectively. Let $A \stackrel{*}{\leq} B$. Then there exist unitary matrices $U \in \mathbb{C}^{m \times m}$ and $V \in \mathbb{C}^{n \times n}$ such that

$$
U^{*} A V=\left(\begin{array}{cc}
D_{a} & 0 \\
0 & 0
\end{array}\right), \quad U^{*} B V=\left(\begin{array}{ccc}
D_{a} & 0 & 0 \\
0 & D & 0 \\
0 & 0 & 0
\end{array}\right),
$$

where both the $a \times a$ matrix $D_{a}$ and the $(b-a) \times(b-a)$ matrix $D$ are real, diagonal, and positive definite (see $[16$, Theorem 2]). In [1, Theorem 5.2.8], it was also shown that

$$
A \stackrel{*}{\leq} B \Longleftrightarrow A^{\dagger} A=B^{\dagger} A, \quad A A^{\dagger}=A B^{\dagger} .
$$

In [17], Wang obtained the following characterizations of the left-star and right-star partial orderings for matrices:

$$
\begin{aligned}
& A^{*} \leq B \Longleftrightarrow r\left[\begin{array}{cc}
B^{*} B & A^{*} A \\
B & A
\end{array}\right]=r(B), \\
& A \leq^{*} B \Longleftrightarrow r\left[\begin{array}{cc}
B B^{*} & A A^{*} \\
B^{*} & A^{*}
\end{array}\right]=r(B), \\
& A \leq B \Longleftrightarrow r\left[\begin{array}{cc}
B^{*} B & A^{*} A \\
B & A
\end{array}\right]=r(B), \\
& r\left[\begin{array}{cc}
B B^{*} & A A^{*} \\
B^{*} & A^{*}
\end{array}\right]=r(B) .
\end{aligned}
$$

Theorem 1. Let $A, B \in \mathbb{C}^{m \times n}$. Then

(i)

$$
A^{*} \leq B \Longleftrightarrow r\left[\begin{array}{cc}
B B^{\dagger} & A A^{\dagger} \\
B^{*} & A^{*}
\end{array}\right]=r(B)
$$

(ii)

$$
A \leq^{*} B \Longleftrightarrow r\left[\begin{array}{cc}
B^{\dagger} B & A^{\dagger} A \\
B & A
\end{array}\right]=r(B)
$$

(iii)

$$
A \stackrel{*}{\leq} B \Longleftrightarrow r\left[\begin{array}{cc}
B^{\dagger} B & A^{\dagger} A \\
B^{*} B & A^{*} A \\
B & A
\end{array}\right]=r(B)
$$

(iv)

$$
A \stackrel{*}{\leq} B \Longleftrightarrow r\left[\begin{array}{cc}
B B^{\dagger} & A A^{\dagger} \\
B B^{*} & A A^{*} \\
B^{*} & A^{*}
\end{array}\right]=r(B) .
$$

Proof. From

$$
\begin{aligned}
r\left[\begin{array}{cc}
B B^{\dagger} & A A^{\dagger} \\
B^{*} & A^{*}
\end{array}\right] & \geq r\left(\left[\begin{array}{cc}
B B^{\dagger} & A A^{\dagger} \\
B^{*} & A^{*}
\end{array}\right]\left[\begin{array}{ll}
B & 0 \\
0 & A
\end{array}\right]\right) \\
& =r\left[\begin{array}{cc}
B & A \\
B^{*} B & A^{*} A
\end{array}\right] \\
& \geq r\left(\left[\begin{array}{cc}
B & A \\
B^{*} B & A^{*} A
\end{array}\right]\left[\begin{array}{cc}
B^{\dagger} & 0 \\
0 & A^{\dagger}
\end{array}\right]\right) \\
& =r\left[\begin{array}{cc}
B B^{\dagger} & A A^{\dagger} \\
B^{*} & A^{*}
\end{array}\right],
\end{aligned}
$$

we have

$$
r\left[\begin{array}{cc}
B^{*} B & A^{*} A \\
B & A
\end{array}\right]=r\left[\begin{array}{cc}
B B^{\dagger} & A A^{\dagger} \\
B^{*} & A^{*}
\end{array}\right] .
$$

Applying (12) gives (i).

In the same way, applying

$$
r\left[\begin{array}{cc}
B^{\dagger} B & A^{\dagger} A \\
B & A
\end{array}\right]=r\left[\begin{array}{cc}
B B^{*} & A A^{*} \\
B^{*} & A^{*}
\end{array}\right]
$$

and (13) gives (ii).

$$
\text { If }
$$

$$
r\left[\begin{array}{cc}
B^{\dagger} B & A^{\dagger} A \\
B^{*} B & A^{*} A \\
B & A
\end{array}\right]=r(B)
$$

then

$$
r\left[\begin{array}{cc}
B B^{*} & A A^{*} \\
B^{*} & A^{*}
\end{array}\right]=r(B), \quad r\left[\begin{array}{cc}
B B^{\dagger} & A A^{\dagger} \\
B^{*} & A^{*}
\end{array}\right]=r(B) .
$$


Applying (i), (ii), and (14), we obtain $A \stackrel{*}{\leq} B$. Conversely, if $A \stackrel{*}{\leq} B$, by using (11) and (14), we have $A^{\dagger} A-B^{\dagger} A=0$, and

$$
\begin{aligned}
r\left[\begin{array}{cc}
B^{*} B & A^{*} A \\
B & A
\end{array}\right] & =r\left[\begin{array}{cc}
0 & A^{\dagger} A-B^{\dagger} A \\
B^{*} B & A^{*} A \\
B & A
\end{array}\right] \\
& =r\left(\left[\begin{array}{ccc}
I_{n} & 0 & B^{\dagger} \\
0 & I_{n} & 0 \\
0 & 0 & I_{m}
\end{array}\right]\left[\begin{array}{cc}
0 & A^{\dagger} A-B^{\dagger} A \\
B^{*} B & A^{*} A \\
B & A
\end{array}\right]\right) \\
& =r\left[\begin{array}{cc}
B^{\dagger} B & A^{\dagger} A \\
B^{*} B & A^{*} A \\
B & A
\end{array}\right], \\
r(B)=r & {\left[\begin{array}{cc}
B^{\dagger} B & A^{\dagger} A \\
B^{*} B & A^{*} A \\
B & A
\end{array}\right] . }
\end{aligned}
$$

Hence, we have (iii).

Similarly, applying $A \stackrel{*}{\leq} B$, (11), and (14), we obtain $A A^{\dagger}-$ $A B^{\dagger}=0, A B^{\dagger}=\left(A B^{\dagger}\right)^{*}=\left(B^{*}\right)^{\dagger} A^{*}$, and

$$
\begin{aligned}
r\left[\begin{array}{cc}
B B^{*} & A A^{*} \\
B^{*} & A^{*}
\end{array}\right] & =r\left[\begin{array}{cc}
0 & A A^{\dagger}-A B^{\dagger} \\
B B^{*} & A A^{*} \\
B^{*} & A^{*}
\end{array}\right] \\
& =r\left(\left[\begin{array}{ccc}
I_{m} & 0 & \left(B^{*}\right)^{\dagger} \\
0 & I_{n} & 0 \\
0 & 0 & I_{n}
\end{array}\right]\left[\begin{array}{cc}
0 & A A^{\dagger}-A B^{\dagger} \\
B B^{*} & A A^{*} \\
B^{*} & A^{*}
\end{array}\right]\right) \\
& =r(B) .
\end{aligned}
$$

Then, we obtain (iv).

In [9, Theorem 2.1], Benítez et al. deduce the characterizations of the left-star, right-star, and star partial orderings for matrices, when at least one of the two involved matrices is EP. When both $A \in \mathbb{C}^{n \times n}$ and $B \in \mathbb{C}^{n \times n}$ are EP matrices, [1, Theorems 5.4.15 and 5.4.2] give the following results:

$A \stackrel{*}{\leq} B \Longleftrightarrow A \leq B, \quad A B^{*}$ and $B^{*} A$ are Hermitian.

$$
A \stackrel{*}{\leq} B \Longleftrightarrow(A B)^{\dagger}=B^{\dagger} A^{\dagger}=A^{\dagger} B^{\dagger}=A^{\dagger^{2}} .
$$

In addition, it was also shown that $A \stackrel{*}{\leq} B$ if and only if $A$ and $B$ have the form

$$
A=U\left[\begin{array}{lll}
T & 0 & 0 \\
0 & 0 & 0 \\
0 & 0 & 0
\end{array}\right] U^{*}, \quad B=U\left[\begin{array}{ccc}
T & 0 & 0 \\
0 & K & 0 \\
0 & 0 & 0
\end{array}\right] U^{*},
$$

where $T \in \mathbb{C}^{r(A) \times r(A)}$ is nonsingular, $K \in \mathbb{C}^{(r(B)-r(A)) \times(r(B)-r(A))}$ is nonsingular, and $U \in \mathbb{C}^{n \times n}$ is unitary (see [1, Theorem 5.4.1])

Based on these results, we consider the characterizations of the star partial ordering for matrices in the set of $\mathbb{C}_{\mathrm{EP}}^{n}$.
Theorem 2. Let $A, B \in \mathbb{C}_{\mathrm{EP}}^{n}, r(B) \geq r(A)$. Then

(

$$
A \leq^{*} B \Longleftrightarrow r\left[\begin{array}{cc}
B & A \\
B^{2} & A^{2}
\end{array}\right]=r(B)
$$

(vi)

$$
A^{*} \leq B \Longleftrightarrow r\left[\begin{array}{cc}
B & B^{2} \\
A & A^{2}
\end{array}\right]=r(B) .
$$

Proof. By $A, B \in \mathbb{C}_{\mathrm{EP}}^{n}$, it is obvious that $A A^{\dagger}=A^{\dagger} A$ and $B B^{\dagger}=B^{\dagger} B$. Then

$$
r\left[\begin{array}{cc}
B & A \\
B^{2} & A^{2}
\end{array}\right]=r(B) \Longleftrightarrow r\left[\begin{array}{cc}
B^{\dagger} B & A^{\dagger} A \\
B & A
\end{array}\right]=r(B) .
$$

Hence, we have (v).

The proof of (vi) is similar to that of (v).

Theorem 3. Let $A, B \in \mathbb{C}_{\mathrm{EP}}^{n}$. Then

(vii)

$$
A \stackrel{*}{\leq} B \Longleftrightarrow r\left[\begin{array}{cc}
B B^{\dagger} & A A^{\dagger} \\
B & A \\
B^{*} & A^{*}
\end{array}\right]=r(B)
$$

(viii)

$$
A \stackrel{*}{\leq} B \Longleftrightarrow r\left[\begin{array}{cc}
B^{*} B & A^{*} A \\
B & A \\
B^{*} & A^{*}
\end{array}\right]=r(B) ;
$$

(ix)

$$
A \stackrel{*}{\leq} B \Longleftrightarrow r\left[\begin{array}{cc}
B & B A \\
B & A B \\
A & A B
\end{array}\right]=r(B)
$$

$(\mathrm{x})$

$$
A \stackrel{*}{\leq} B \Longleftrightarrow r\left[\begin{array}{ll}
B & B A^{\dagger} \\
B & A^{\dagger} B \\
A & A^{\dagger} B
\end{array}\right]=r(B)
$$

(xi)

$$
A \stackrel{*}{\leq} B \Longleftrightarrow r\left[\begin{array}{ll}
B & B A^{*} \\
B & A^{*} B \\
A & A^{*} B
\end{array}\right]=r(B)
$$

Proof. By $A, B \in \mathbb{C}_{\mathrm{EP}}^{n}$, it is obvious that $A A^{\dagger}=A^{\dagger} A$ and $B B^{\dagger}=B^{\dagger} B$. Applying (i), (ii), and the rank equality in (vii) we obtain

$$
r\left[\begin{array}{cc}
B^{\dagger} B & A^{\dagger} A \\
B & A
\end{array}\right]=r(B), \quad r\left[\begin{array}{cc}
B B^{\dagger} & A A^{\dagger} \\
B^{*} & A^{*}
\end{array}\right]=r(B) ;
$$


that is, $A \stackrel{*}{\leq} B$. Conversely, suppose that $A \stackrel{*}{\leq} B$. Applying $A-A A^{\dagger} B=0$ and $B^{*} B B^{\dagger}=B^{*}$, we obtain

$$
\begin{aligned}
r(B) & =r\left[\begin{array}{cc}
B B^{\dagger} & A A^{\dagger} \\
B & A
\end{array}\right]=r\left[\begin{array}{cc}
B B^{\dagger} & A A^{\dagger} \\
B & A \\
0 & A^{*}-B^{*} A A^{\dagger}
\end{array}\right] \\
& =r\left[\begin{array}{cc}
B B^{\dagger} & A A^{\dagger} \\
B & A \\
B^{*} & A^{*}
\end{array}\right] .
\end{aligned}
$$

Applying (11), we obtain $B^{*} B B^{\dagger} B=B^{*} B$ and $B^{*} B A^{\dagger} A=$ $A^{*} A$ and also $\left(B^{*} B\right)^{\dagger} B^{*} B=B^{\dagger} B$ and $\left(B^{*} B\right)^{\dagger} A^{*} A=A^{\dagger} A$. Then

$$
\begin{aligned}
& r\left[\begin{array}{cc}
B B^{\dagger} & A A^{\dagger} \\
B & A \\
B^{*} & A^{*}
\end{array}\right]=r\left[\begin{array}{cc}
B^{\dagger} B & A^{\dagger} A \\
B & A \\
B^{*} & A^{*}
\end{array}\right] \\
& \geq r\left(\left[\begin{array}{ccc}
B^{*} B & 0 & 0 \\
0 & I_{n} & 0 \\
0 & 0 & I_{n}
\end{array}\right]\left[\begin{array}{cc}
B^{\dagger} B & A^{\dagger} A \\
B & A \\
B^{*} & A^{*}
\end{array}\right]\right) \\
& =r\left[\begin{array}{cc}
B^{*} B & A^{*} A \\
B & A \\
B^{*} & A^{*}
\end{array}\right] \\
& \geq r\left(\left[\begin{array}{ccc}
\left(B^{*} B\right)^{\dagger} & 0 & 0 \\
0 & I_{n} & 0 \\
0 & 0 & I_{n}
\end{array}\right]\left[\begin{array}{cc}
B^{*} B & A^{*} A \\
B & A \\
B^{*} & A^{*}
\end{array}\right]\right) \\
& =r\left[\begin{array}{cc}
B^{\dagger} B & A^{\dagger} A \\
B & A \\
B^{*} & A^{*}
\end{array}\right]
\end{aligned}
$$

that is,

$$
r\left[\begin{array}{cc}
B B^{\dagger} & A A^{\dagger} \\
B & A \\
B^{*} & A^{*}
\end{array}\right]=r\left[\begin{array}{cc}
B^{*} B & A^{*} A \\
B & A \\
B^{*} & A^{*}
\end{array}\right]
$$

Hence, we have (viii).

Suppose that $A \stackrel{*}{\leq} B$. Since $A, B \in \mathbb{C}_{\mathrm{EP}}^{n}$, applying (27), it is easy to check the rank equality in (ix). Conversely, under the rank equality in (ix), we have

$$
\begin{gathered}
r\left[\begin{array}{ll}
B & B A \\
B & A B
\end{array}\right]=r\left[\begin{array}{cc}
B & B A \\
0 & A B-B A
\end{array}\right]=r(B) \Longrightarrow A B=B A, \\
r\left[\begin{array}{ll}
B & B A \\
A & A B
\end{array}\right]=r\left[\begin{array}{cc}
B & 0 \\
A & A B-A A^{2}
\end{array}\right]=r(B) \Longrightarrow A B=A^{2} .
\end{gathered}
$$

Since $A$ is EP, there exists a unitary matrix $U_{1} \in \mathbb{C}^{n \times n}$ and a nonsingular matrix $T \in \mathbb{C}^{r(A) \times r(A)}$ such that

$$
A=U_{1}\left[\begin{array}{ll}
T & 0 \\
0 & 0
\end{array}\right] U_{1}^{*}
$$

Correspondingly denote $P^{-1} B P$ by

$$
B=U_{1}\left[\begin{array}{ll}
B_{1} & B_{2} \\
B_{3} & B_{4}
\end{array}\right] U_{1}^{*},
$$

where $B_{1} \in \mathbb{C}^{r(A) \times r(A)}$. It follows that

$$
\left[\begin{array}{cc}
T B_{1} & T B_{2} \\
0 & 0
\end{array}\right]=\left[\begin{array}{cc}
B_{1} T & 0 \\
B_{3} T & 0
\end{array}\right], \quad\left[\begin{array}{cc}
T B_{1} & T B_{2} \\
0 & 0
\end{array}\right]=\left[\begin{array}{cc}
T^{2} & 0 \\
0 & 0
\end{array}\right] .
$$

Since $T$ is a unitary matrix,

$$
B_{1}=T, \quad B_{2}=0, \quad B_{3}=0 .
$$

Thus

$$
B=U\left[\begin{array}{cc}
T & 0 \\
0 & B_{4}
\end{array}\right] U^{*}
$$

Since $B$ is EP, $B_{4}$ is EP, and there exists a unitary matrix $U_{2} \in \mathbb{C}^{(n-r(A)) \times(n-r(A))}$ and a nonsingular matrix $K \in$ $\mathbb{C}^{(r(B)-r(A)) \times(r(B)-r(A))}$ such that

$$
B_{4}=U_{2}\left[\begin{array}{cc}
K & 0 \\
0 & 0
\end{array}\right] U_{2}^{*}
$$

Write

$$
U=U_{1}\left[\begin{array}{cc}
0 & 0 \\
0 & U_{2}
\end{array}\right]
$$

Then $A$ and $B$ have the form

$$
A=U\left[\begin{array}{lll}
T & 0 & 0 \\
0 & 0 & 0 \\
0 & 0 & 0
\end{array}\right] U^{*}, \quad B=U\left[\begin{array}{ccc}
T & 0 & 0 \\
0 & K & 0 \\
0 & 0 & 0
\end{array}\right] U^{*} \text {. }
$$

Applying (27), we have $A \stackrel{*}{\leq} B$.

The proofs of (x) and (xi) are similar to that of (ix).

\section{The Sharp Partial Ordering}

Let $A, B \in \mathbb{C}_{\mathrm{GP}}^{n}$ with ranks $a$ and $b$, respectively. It is well known that

$$
A \leq^{\#} B \Longleftrightarrow A^{2}=A B=B A .
$$

In addition, $A \leq^{\#} B$ if and only if $A$ and $B$ can be written as

$$
A=P\left[\begin{array}{ccc}
E & 0 & 0 \\
0 & 0 & 0 \\
0 & 0 & 0
\end{array}\right] P^{-1}, \quad B=P\left[\begin{array}{ccc}
E & 0 & 0 \\
0 & E^{\prime} & 0 \\
0 & 0 & 0
\end{array}\right] P^{-1}
$$

where $E \in \mathbb{C}^{a \times a}$ is nonsingular, $E^{\prime} \in \mathbb{C}^{(b-a) \times(b-a)}$ is nonsingular, and $P \in \mathbb{C}^{n \times n}$ is nonsingular (see [18]).

In Theorem 4, we give one characterization of the sharp partial ordering by using one rank equality.

Theorem 4. Let $A, B \in \mathbb{C}_{\mathrm{GP}}^{n}$. Then

$$
A \leq^{\#} B \Longleftrightarrow r\left[\begin{array}{cc}
A & B A \\
A B & A B A
\end{array}\right]=r(A B A) .
$$


Proof. Let $A$ have the core-nilpotent decomposition (see [19, Exercise 5.10.12])

$$
A=P\left[\begin{array}{ll}
\Sigma & 0 \\
0 & 0
\end{array}\right] P^{-1}
$$

with nonsingular matrices $\Sigma \in \mathbb{C}^{r(A) \times r(A)}$ and $P \in \mathbb{C}^{n \times n}$. Correspondingly denote $P^{-1} B P$ by

$$
P^{-1} B P=\left[\begin{array}{ll}
B_{1} & B_{2} \\
B_{3} & B_{4}
\end{array}\right]
$$

where $B_{1} \in \mathbb{C}^{r(A) \times r(A)}$. It follows that

$$
\begin{aligned}
& r(A B A)=r\left(\Sigma B_{1} \Sigma\right), \\
& r\left[\begin{array}{cc}
A & B A \\
A B & A B A
\end{array}\right]= r\left[\begin{array}{ccc}
\Sigma & 0 & B_{1} \Sigma \\
0 & 0 & B_{3} \Sigma \\
\Sigma B_{1} & \Sigma B_{2} & \Sigma B_{1} \Sigma
\end{array}\right] \\
& r\left[\begin{array}{ccc}
\Sigma & 0 & 0 \\
0 & 0 & B_{3} \Sigma \\
0 & \Sigma B_{2} & \Sigma B_{1} \Sigma-\Sigma B_{1} \Sigma^{-1} B_{1} \Sigma
\end{array}\right] \\
& r(\Sigma)+r\left[\begin{array}{cc}
0 & B_{3} \Sigma \\
\Sigma B_{2} & \Sigma B_{1} \Sigma-\Sigma B_{1} \Sigma^{-1} B_{1} \Sigma
\end{array}\right] .
\end{aligned}
$$

Applying (54) to the rank equality in (51), we obtain

$$
r\left[\begin{array}{cc}
0 & B_{3} \Sigma \\
\Sigma B_{2} & \Sigma B_{1} \Sigma-\Sigma B_{1} \Sigma^{-1} B_{1} \Sigma
\end{array}\right]+r(\Sigma)=r\left(\Sigma B_{1} \Sigma\right) .
$$

Hence $r\left(\Sigma B_{1} \Sigma\right)=r(\Sigma), \Sigma B_{2}=0, B_{3} \Sigma=0$, and $\Sigma B_{1} \Sigma=$ $\Sigma B_{1} \Sigma^{-1} B_{1} \Sigma$. Since $\Sigma \in \mathbb{C}^{r(A) \times r(A)}$ is invertible and $B_{1} \in$ $\mathbb{C}^{r(A) \times r(A)}$, it follows immediately that

$$
r\left(B_{1}\right)=r(\Sigma), \quad B_{3}=0, \quad B_{2}=0, \quad B_{1}=\Sigma .
$$

Therefore

$$
B=P\left[\begin{array}{cc}
\Sigma & 0 \\
0 & B_{4}
\end{array}\right] P^{-1}
$$

Applying

$$
\begin{aligned}
A^{2} & =P\left[\begin{array}{cc}
\Sigma^{2} & 0 \\
0 & 0
\end{array}\right] P^{-1} \\
& =P\left[\begin{array}{cc}
\Sigma & 0 \\
0 & 0
\end{array}\right] P^{-1} P\left[\begin{array}{cc}
\Sigma & 0 \\
0 & B_{4}
\end{array}\right] P^{-1} \\
& =A B \\
& =P\left[\begin{array}{cc}
\Sigma & 0 \\
0 & B_{4}
\end{array}\right] P^{-1} P\left[\begin{array}{cc}
\Sigma & 0 \\
0 & 0
\end{array}\right] P^{-1} \\
& =B A,
\end{aligned}
$$

and (49), we obtain that $A \leq^{\#} B$.

Conversely, it is a simple matter.

\section{Conflict of Interests}

The authors declare that there is no conflict of interests regarding the publication of this paper.

\section{Acknowledgments}

The authors would like to thank the referees for their helpful comments and suggestions. The work of the first author was supported in part by the Foundation of Anhui Educational Committee (Grant no. KJ2012B175) and the National Natural Science Foundation of China (Grant no. 11301529). The work of the second author was supported in part by the Foundation of Anhui Educational Committee (Grant no. KJ2013B256).

\section{References}

[1] S. K. Mitra, P. Bhimasankaram, and S. B. Malik, Matrix Partial Orders, Shorted Operators and Applications, World Scientific, Singapore, 2010.

[2] G. Wang, Y. Wei, and S. Qiao, Generalized Inverses: Theory and Computations, Science Press, Beijing, China, 2004.

[3] R. E. Hartwig, "How to partially order regular elements," Mathematica Japonica, vol. 25, no. 1, pp. 1-13, 1980.

[4] K. S. S. Nambooripad, "The natural partial order on a regular semigroup," Proceedings of the Edinburgh Mathematical Society, vol. 23, no. 3, pp. 249-260, 1980.

[5] M. P. Drazin, "Natural structures on semigroups with involution," Bulletin of the American Mathematical Society, vol. 84, no. 1, pp. 139-141, 1978.

[6] J. K. Baksalary and S. K. Mitra, "Left-star and right-star partial orderings," Linear Algebra and Its Applications, vol. 149, pp. 7389, 1991.

[7] S. K. Mitra, "On group inverses and the sharp order," Linear Algebra and Its Applications, vol. 92, pp. 17-37, 1987.

[8] J. K. Baksalary, O. M. Baksalary, and X. Liu, "Further properties of the star, left-star, right-star, and minus partial orderings," Linear Algebra and Its Applications, vol. 375, pp. 83-94, 2003.

[9] J. Benítez, X. Liu, and J. Zhong, "Some results on matrix partial orderings and reverse order law," Electronic Journal of Linear Algebra, vol. 20, pp. 254-273, 2010.

[10] J. Groß, "Remarks on the sharp partial order and the ordering of squares of matrices," Linear Algebra and Its Applications, vol. 417, no. 1, pp. 87-93, 2006.

[11] Z.-J. Bai and Z.-Z. Bai, "On nonsingularity of block two-by-two matrices," Linear Algebra and Its Applications, vol. 439, no. 8, pp. 2388-2404, 2013.

[12] D. Chu, Y. S. Hung, and H. J. Woerdeman, "Inertia and rank characterizations of some matrix expressions," SIAM Journal on Matrix Analysis and Applications, vol. 31, no. 3, pp. 1187-1226, 2009.

[13] Y. Liu and Y. Tian, "A simultaneous decomposition of a matrix triplet with applications," Numerical Linear Algebra with Applications, vol. 18, no. 1, pp. 69-85, 2011.

[14] H. Wang, "The minimal rank of $A-B X$ with respect to Hermitian matrix," Applied Mathematics and Computation, vol. 233, pp. 55-61, 2014.

[15] Q.-W. Wang and Z.-H. He, "Solvability conditions and general solution for mixed Sylvester equations," Automatica, vol. 49, no. 9, pp. 2713-2719, 2013. 
[16] R. E. Hartwig and G. P. H. Styan, "On some characterizations of the "star" partial ordering for matrices and rank subtractivity," Linear Algebra and Its Applications, vol. 82, pp. 145-161, 1986.

[17] H. X. Wang, "Rank characterizations of some matrix partial orderings," Journal of East China Normal University, no. 5, pp. 5-11, 2011.

[18] Z. J. Wang and X. J. Liu, "On three partial orderings of matrices," Journal of Mathematical Study, vol. 36, no. 1, pp. 75-81, 2003.

[19] C. D. Meyer, Matrix Analysis and Applied Linear Algebra, Society for Industrial and Applied Mathematics, Philadelphia, Pa, USA, 2000. 


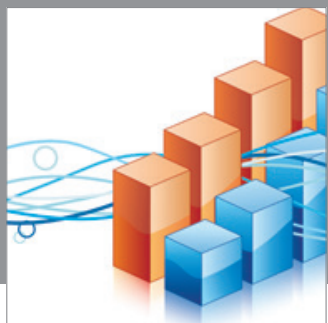

Advances in

Operations Research

mansans

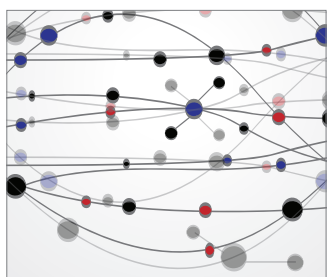

The Scientific World Journal
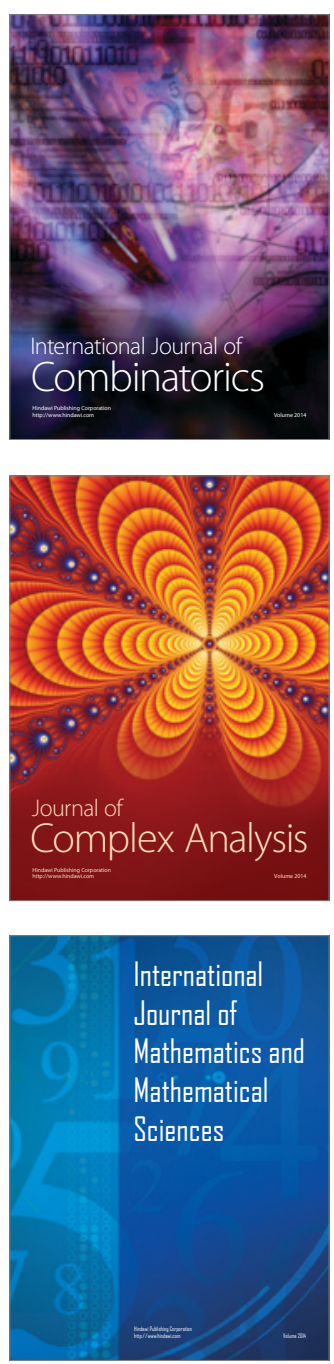
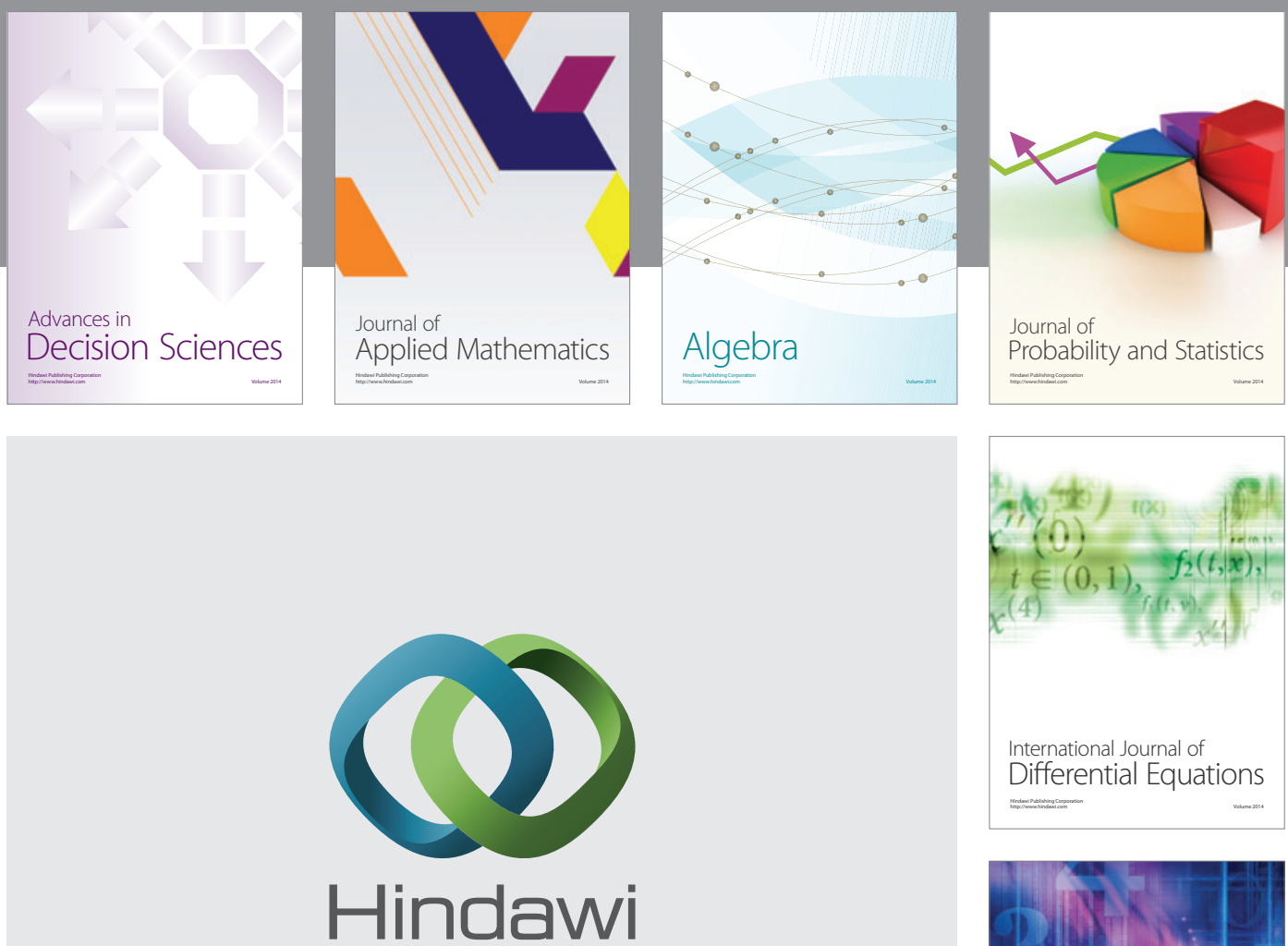

Submit your manuscripts at http://www.hindawi.com
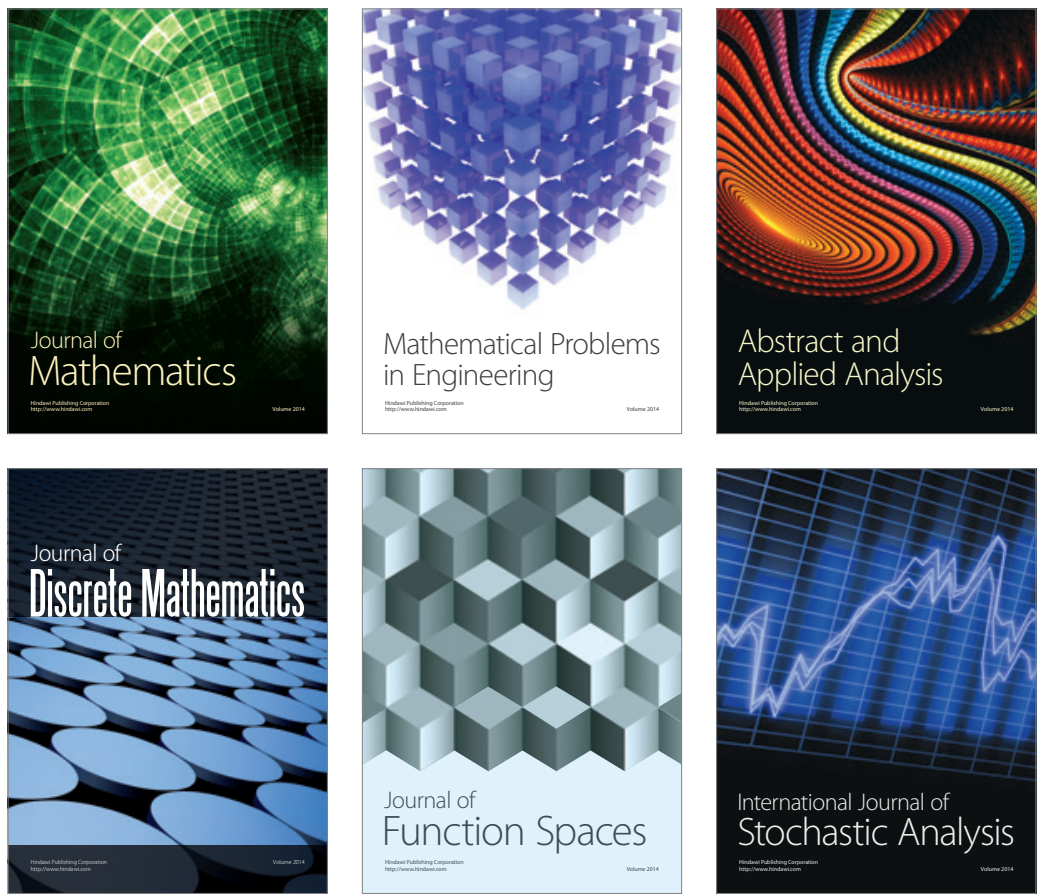

Journal of

Function Spaces

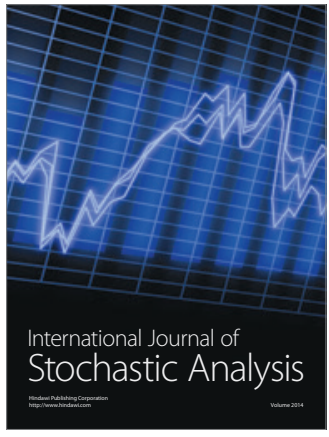

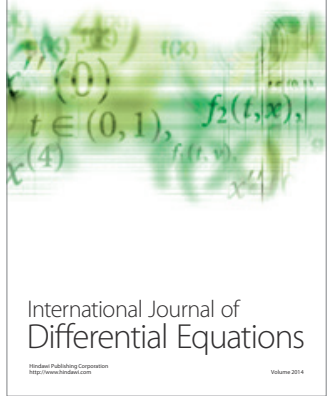
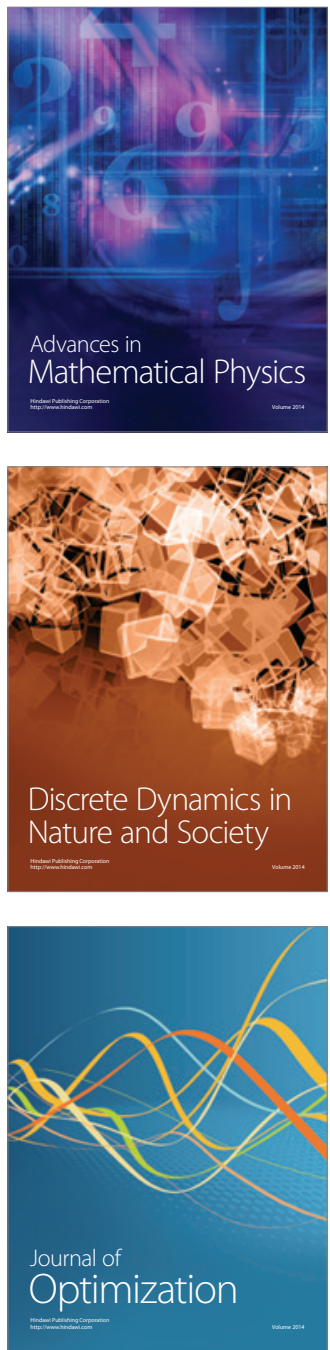